\title{
Interaction of $\alpha$-taxilin Localized on Intracellular Components with the Microtubule Cytoskeleton
}

\author{
Yukimi Horii $^{1}$, Satoru Nogami ${ }^{\sharp \#}$, Yoji Kawano ${ }^{1 \$}$, Takako Kaneko-Kawano ${ }^{1 \&}$, Natsuko Ohtomo ${ }^{2}$, \\ Tomoaki Tomiya ${ }^{2}$, and Hiromichi Shirataki ${ }^{*}$ \\ ${ }^{1}$ Department of Molecular and Cell Biology, Graduate School of Medicine, Dokkyo Medical University, \\ 880 Kitakobayashi, Mibu, Tochigi 321-0293, Japan, ${ }^{2}$ Department of Gastroenterology, Graduate School of \\ Medicine, The University of Tokyo, 7-3-1 Hongo, Bunkyo-ku, Tokyo 113-8655, Japan
}

\begin{abstract}
$A B S T R A C T$. Intracellular vesicle traffic plays an essential role in the establishment and maintenance of organelle identity and biosynthetic transport. We have identified $\alpha$-taxilin as a binding partner of the syntaxin family, which is involved in intracellular vesicle traffic. Recently, we have found that $\alpha$-taxilin is over-expressed in malignant tissues including hepatocellular carcinoma and renal cell carcinoma. However, a precise role of $\alpha$ taxilin in intracellular vesicle traffic and carcinogenesis remains unclear. Then, we first investigated here the intracellular distribution of $\alpha$-taxilin in Hela cells. Immunofluorescence studies showed that $\alpha$-taxilin distributes throughout the cytoplasm and exhibits a tubulo-vesicular pattern. Biochemical studies showed that $\alpha$-taxilin is abundantly localized on intracellular components as a peripheral membrane protein. Moreover, we found that $\alpha$-taxilin distributes in microtubule-dependent and syntaxin-independent manners, that $\alpha$-taxilin directly binds to polymerized tubulin in vitro, and that $N$-ethylmaleimide but not brefeldin $A$ affects the intracellular distribution of $\alpha$-taxilin. These results indicate that $\alpha$-taxilin is localized on intracellular components in a syntaxin-independent manner and that the $\alpha$-taxilin-containing intracellular components are associated with the microtubule cytoskeleton and suggest that $\alpha$-taxilin functions as a linker protein between the $\alpha$-taxilin-containing intracellular components and the microtubule cytoskeleton.
\end{abstract}

Key words: Vesicle transport/ $\alpha$-taxilin/syntaxin/SNARE/tubulin

\footnotetext{
*To whom correspondence should be addressed: Hiromichi Shirataki, Department of Molecular and Cell Biology, Graduate School of Medicine, Dokkyo Medical University, 880 Kitakobayashi, Mibu, Tochigi 321-0293, Japan.

Tel: +81-282-87-2140, Fax: +81-282-86-3533

${ }^{\text {\# } P r e s e n t ~ a d d r e s s: ~ S a t o r u ~ N o g a m i, ~ D e p a r t m e n t ~ o f ~ I n t e g r a t e d ~ B i o s c i e n c e s, ~}$ Graduate School of Frontier Sciences, University of Tokyo, 5-1-5 Kashiwanoha, Kashiwa, Chiba 277-8562, Japan.

\$ Present address: Yoji Kawano, Laboratory of Plant Molecular Genetics, Nara Institute of Science and Technology, 8916-5 Takayama, Ikoma, Nara 630-0101, Japan.

\& Present address: Takako Kaneko-Kawano, College of Pharmaceutical Sciences, Ritsumeikan University, 1-1-1 Noji Higashi, Kusatsu, Shiga 5258577, Japan.

Abbreviations: SNARE, soluble $N$-ethylmaleimide-sensitive factor attachment protein receptor; $\mathrm{v}$-SNARE, vesicle SNARE; t-SNARE, target SNARE; SNAP-25, synaptosomal-associated protein of $25 \mathrm{kDa}$; MBP, microtubule-binding protein; NEM, $N$-ethylmaleimide; TfR, transfferin receptor; GST, glutathione-S-transferase; DMEM, Dulbecco's modified Eagle's medium; PBS, phosphate buffered saline; siRNA, small interfering RNA; NSF, NEM-sensitive fusion factor; PAGE, polyacrylamide gel electrophoresis; ER, endoplasmic reticulum; TGN, trans-Golgi network; NAC, nascent polypeptide-associated complex; ATF4, activating transcription factor 4; FIAT, factor inhibiting ATF4-mediated transcription.
}

\section{Introduction}

Intracellular vesicle traffic is an essential process for the synthesis and processing of proteins and lipids as well as the maintenance of the compartmentalization of cells (Rothman, 1994). Moreover, increasing evidence indicates that intracellular vesicle traffic is involved in the processes of carcinogenesis, tumor progression, and metastasis in various tumors (Bascom et al., 2005; Hashimoto et al., 2004; Miyata et al., 2004; Stromberg et al., 2009). Intracellular vesicle traffic includes budding of vesicles from a donor membrane, delivery of the vesicles along the cytoskeleton to the acceptor membrane, and fusion of the vesicles with the acceptor membrane. Available evidence indicates that the vesicles deliver their cargo at the acceptor membrane through a strict succession of three interdependent processes composed of tethering, docking, and fusion and that all of the processes are spatially and temporally regulated to achieve cargo delivery to the correct destination (Cai et al., 2007a). Soluble $N$-ethylmaleimide-sensitive factor attachment protein receptors (SNAREs) play a central 
role in these three processes (Rothman, 1994). SNAREs are a superfamily of integral membrane proteins with $\alpha$-helical motif. They are composed of vesicle SNARE (v-SNARE) resided on vesicles and target SNARE (t-SNARE) resided on the acceptor membrane. V-SNARE forms a stable complex (a so-called trans-SNARE complex) with cognate t-SNAREs, resulting that the vesicles are tethered to, dock to and subsequently fuse with the acceptor membrane. A unique set of SNAREs is localized on distinct membrane compartments. Therefore, it has been originally proposed that the complex formation of $\mathrm{v}-\mathrm{SNARE}$ with cognate $\mathrm{t}$ SNARE provides the specificity of vesicle targeting to the acceptor membrane. After then, however, a growing body of evidence indicates that in addition to SNAREs, other molecules are needed to mediate the specificity of the vesicle targeting (Cai et al., 2007a; Jahn and Scheller, 2006). These molecules (so called tethering components) include Exocyst, small GTPases of the Ypt/Rab and Arf families, coiled-coil proteins, and large multi-protein assemblies recently termed quatrefoil tethering complexes (Behnia and Munro, 2005; Brocker et al., 2010; Gillingham and Munro, 2003; Sztul and Lupashin, 2006). The tethering components are thought to act before the trans-SNARE complex formation and provide an initial interaction between vesicles and the acceptor membrane.

The syntaxin family composed of over a dozen members belongs to t-SNAREs (Bennett et al., 1993). Each of the syntaxin family members is localized on distinct membrane compartments. Syntaxin-1s, $-2,-3$, and -4 are predominantly localized on the plasma membrane and the other members are predominantly localized on the restricted intracellular organelles (Chen and Scheller, 2001). The former are involved in transport of the vesicles delivered to the plasma membrane, whereas the latter are involved in vesicle transport between intracellular organelles. Accumulating evidence indicates that the syntaxin family is a core molecule on the formation of the trans-SNARE complex. Moreover, it has been revealed that several of the syntaxinbinding proteins function as tethering components in intracellular vesicle traffic (Cai et al., 2007a). For example, Rab4, a small GTPase of the Ypt/Rab and Arf families, binds to syntaxin-4 in a guanine nucleotide-dependent manner (Li et al., 2001). p115, a coiled-coil protein, interacts with syntaxin-5 and thereby stimulates the specific assembly of the trans-SNARE complex containing syntaxin5 (Sapperstein et al., 1995; Shorter et al., 2002). Taken together, the syntaxin family is thought to play an important role not only in the formation of the trans-SNARE complex but also in the specificity of the vesicle targeting.

We have previously identified the taxilin family as a binding partner of the syntaxin family (Nogami et al., 2003a) and, moreover, recently revealed that the expression of $\alpha$-taxilin is correlated with the histological grade and proliferative activity of hepatocellular carcinoma and the metastatic and invasive potential of renal cell carcinoma
(Mashidori et al., 2011; Ohtomo et al., 2010). Therefore, the taxilin family is increasingly appreciated as an important molecule in clinical and pathophysiological fields. In mammals, the taxilin family is composed of at least three members, $\alpha-, \beta$-, and $\gamma$-taxilins (Nogami et al., 2004). $\alpha$ and $\gamma$-Taxilins are ubiquitously expressed but $\beta$-taxilin is abundantly expressed in the skeletal muscle and heart. The taxilin family members share an extraordinarily long coiledcoil region homologous to that of Uso1, a yeast coiled-coil protein homologue of p115 (Nogami et al., 2003a). The coiled-coil region is responsible for the interaction of the taxilin family with the syntaxin family (Nogami et al., 2004). The taxilin family members interact with syntaxin1a, -3 , and -4 localized on the plasma membrane but not with syntaxin-7 or -8 localized on the intracellular organelles, implying that the taxilin family is involved in transport of the vesicles delivered to the plasma membrane (Nogami et al., 2003a). However, since $\alpha$ - and $\gamma$-taxilins, and $\beta$-taxilin more favorably interact with syntaxin- 4 and -1a, respectively, it is considered possible that $\beta$-taxilin has different functions from the other taxilin family members in intracellular vesicle traffic (Nogami et al., 2004). Until now, a precise role of the taxilin family in intracellular vesicle traffic, especially the formation of the trans-SNARE complex, remains to be clarified. However, taken together with our earlier reports that $\alpha$-taxilin is able to interact with syntaxin-1a free of vesicle-associated membrane protein-2, synaptosomal-associated protein of $25 \mathrm{kDa}$ (SNAP-25), and Munc18, that both SNAP-25 and Munc18 inhibit the interaction of $\alpha$-taxilin with syntaxin-1a, and that the extraordinarily long coiled-coil region of the taxilin family is homologous to that of Uso1, a yeast tethering component, it is thought that the taxilin family may function as a tethering component before the formation of the trans-SNARE complex (Nogami et al., 2003b).

The microtubule cytoskeleton plays a fundamental role in the dynamic organization of cytoplasmic organelles through controlling intracellular membrane traffic (Kelly, 1990). A large number of microtubule-binding proteins (MBPs) have been identified and the molecular mechanisms by which these factors regulate intracellular membrane traffic along the microtubule cytoskeleton have been increasingly well understood. The MBPs are divided into two groups; motor and non-motor MBPs. Motor MBPs are composed of kinesin and cytoplasmic dynein, which are known to be responsible for directed movement of organelles along the microtubule cytoskeleton. Non-motor MBPs are composed of a variety of proteins, which have abilities to regulate the assembly or disassembly of microtubules, the forcegenerating activity of motor MBPs, and/or the interaction of organelles with the microtubule cytoskeleton. Accumulating evidence indicates that several of the molecules which have been initially identified as SNARE-related molecules also function as non-motor MBPs (Cai et al., 2007b; Fujiwara et al., 1997; Honda et al., 2002; Su et al., 2004; 
Wang et al., 2004), raising the possibility that the SNARErelated non-motor MBPs are sequentially involved in microtubule rearrangement and intracellular vesicle traffic.

To find clues to the function of $\alpha$-taxilin in intracellular vesicle traffic, we here attempted to determine the intracellular distribution of $\alpha$-taxilin in Hela cells. $\alpha$-Taxilin was abundantly localized as a peripheral membrane protein on the intracellular components which were distributed throughout the cytoplasm in microtubule-dependent and syntaxin-independent manners. Although $\alpha$-taxilin has no microtubule-binding domain characterized so far, surprisingly $\alpha$-taxilin directly bound to polymerized tubulin. The intracellular distribution of $\alpha$-taxilin was affected by treatment with $N$-ethylmaleimide (NEM), which inhibits intracellular vesicle traffic from the trans-Golgi network to the plasma membrane. Taken together with our earlier report that $\alpha$-taxilin binds to syntaxin-3 and -4 , both of which are mainly localized on the plasma membrane (Nogami et al., 2003a), we propose here the attractive possibility that $\alpha$-taxilin mediates the transport of $\alpha$-taxilincontaining intracellular components along the microtubule cytoskeleton to the plasma membrane. The findings of our present study provide important insights into the function of $\alpha$-taxilin in intracellular vesicle traffic.

\section{Materials and Methods}

\section{Reagents}

An anti- $\alpha$-taxilin polyclonal antibody was prepared as previously described (Yoshida et al., 2005). His ${ }_{6}-\alpha$-taxilin was prepared as previously described (Nogami et al., 2003b). Antibodies against the following proteins or peptide were purchased: GM130, syntaxin-4, syntaxin-6, BiP, Rab11, and calnexin from BD Biosciences (San Diego, CA, USA); LAMP1 and transferrin receptor (TfR) from Invitrogen Corp. (Carlsbad, CA, USA); $\alpha$-tubulin from Sigma (St Louis, MO, USA); KDEL from Enzo Life Sciences Inc. (Farmingdale, NY, USA); syntaxin-3 from Synaptic systems (Göttingen, Germany); syntaxin-18 from abcam (Cambridge, MA, USA); and glutathione- $S$-transferase (GST) from MBL (Nagoya, Japan). Bovine brain tubulin was from Cytoskeleton (Denver, CO, USA). Other materials were obtained from commercial sources.

\section{Cell culture, transfection, and immunofluorescence}

Hela cells were cultured in a humidified atmosphere of $5 \% \mathrm{CO}_{2}$ and 95\% air in Dulbecco's modified Eagle's medium (DMEM) containing 10\% fetal calf serum (Invitrogen Corp.), 100 units $/ \mathrm{ml}$ penicillin, and $100 \mu \mathrm{g} / \mathrm{ml}$ streptomycin at $37^{\circ} \mathrm{C}$. Transient transfection of pEGFP- and pcDNA-Myc- $\alpha$-taxilins into Hela cells was performed by use of Lipofectamine ${ }^{\mathrm{TM}} 2000$ reagent (Invitrogen Corp.) according to the manufacturer's instructions. The transfected cells were used for experiments using NEM at $24 \mathrm{~h}$ posttransfection. For immunofluorescence, cells were grown on glass coverslips, washed three times briefly in phosphate buffered saline (PBS), and fixed with 3.7\% formaldehyde in PBS for $10 \mathrm{~min}$. The fixed cells were permeabilized with $0.1 \%$ Triton X-100 in PBS for 10 min. After being blocked with PBS containing 10\% normal goat serum and $1 \%$ bovine serum albumin for $1 \mathrm{~h}$, the cells were treated with the first antibody in PBS containing $10 \%$ normal goat serum and $1 \%$ bovine serum albumin for $1 \mathrm{~h}$. The cells were washed three times in PBS, followed by incubation with the second antibody in PBS containing 10\% normal goat serum and $1 \%$ bovine serum albumin for $1 \mathrm{~h}$. After being washed three times in PBS, the cells were examined by use of an Axiovert 200 fluorescence microscope with an Apotome device for optical sectioning (Carl Zeiss, Thornwood, NY, USA).

\section{Small interfering RNA (siRNA) treatment}

Hela cells were transfected with $10 \mathrm{nM}$ siRNA oligo duplex against human $\alpha$-taxilin (5'-UGCAUCAGCAACGUGAUCUCCUUCC-3'), human syntaxin-3 (5'-CCAACAACGUCCGGAACAAACUGAA-3'), human syntaxin-4 (5'-GGCACAGUGAGAUCCAGCAGCUUGA-3'), human syntaxin-18 (5'-CAGGACCGCUGUUUUGGAUUU-3'), or luciferase GL2 (5'-CGUACGCGGAAUACUUCGATT-3') using Lipofectamine ${ }^{\mathrm{TM}}$ RNAiMAX (Invitrogen Corp.) according to the manufacturer's instructions. In one set of experiments, the cells were collected, homogenized, and subfractionated by centrifugation as described below at 2 days after transfection. In another set of experiments, the cells were trypsinized and replated on glass coverslips at 2 days after transfection. Then, at one day after being replated, the cells were fixed as described above and used for immunofluorescence analysis.

\section{Treatment with nocodazole, NEM, and brefeldin A}

To depolymerize microtubules, Hela cells were incubated in DMEM containing $10 \%$ fetal calf serum, 100 units $/ \mathrm{ml}$ penicillin, and $100 \mu \mathrm{g} / \mathrm{ml}$ streptomycin for $30 \mathrm{~min}$ at $4^{\circ} \mathrm{C}$ and further incubated in the presence of $1 \mu \mathrm{M}$ nocodazole for $30 \mathrm{~min}$ at $37^{\circ} \mathrm{C}$. To repolymerize microtubules, the cells treated with nocodazole as described above were washed twice in PBS and further incubated in the absence of nocodazole for the indicated periods at $37^{\circ} \mathrm{C}$. To inactivate NEM-sensitive fusion factor (NSF), a membrane fusion component, Hela cells were incubated with $0.5 \mathrm{mM}$ NEM in PBS for $15 \mathrm{~min}$ at $4^{\circ} \mathrm{C}$. After then, the cells were washed twice in PBS and further incubated in DMEM in the absence of NEM for $3 \mathrm{~h}$ at $37^{\circ} \mathrm{C}$. To inhibit the anterograde transport from the endoplasmic reticulum to the Golgi apparatus, Hela cells were incubated with $1 \mu \mathrm{g} / \mathrm{ml}$ of brefeldin A in DMEM containing $10 \%$ fetal calf serum for $90 \mathrm{~min}$ at $37^{\circ} \mathrm{C}$.

\section{Subcellular fractionation and depletion of $\alpha$-taxilin-containing intracellular components}

All the following procedures were performed at $4^{\circ} \mathrm{C}$. Approximately $80 \%$ confluent Hela cells (four $10-\mathrm{cm}$ dishes) were washed twice in Buffer A (10 mM Tris/ $\mathrm{HCl}$ at $\mathrm{pH} 7.5,1 \mathrm{mM}$ EDTA, and 
$0.25 \mathrm{M}$ sucrose). The cells were collected, suspended in $2 \mathrm{ml}$ of Buffer A containing $10 \mu \mathrm{M} p$-amidinophenylmethansulfonyl fluoride and $10 \mu \mathrm{M}$ leupeptin, and homogenized with 15 strokes in a ball homogenizer (clearance: $0.012 \mathrm{~nm}$ ). The homogenate was centrifuged at $1,000 \times \mathrm{g}$ for $10 \mathrm{~min}$ and the supernatant was used as the post-nuclear fraction. An aliquot of the supernatant was further centrifuged at $100,000 \times \mathrm{g}$ for $1 \mathrm{~h}$ to separate the cytosol and membrane fractions. The membrane fraction was suspended in comparable volume of Buffer A and the sample was layered onto a $2-$ $20 \%$ OptiPrep (Invitrogen Corp.) gradient in Buffer A, followed by centrifugation at $130,000 \times g$ for $3 \mathrm{~h}$. After centrifugation, twenty fractions $(0.65 \mathrm{ml}$ each) were collected from the top to bottom. For depletion of $\alpha$-taxilin-containing intracellular components, the membrane fraction was suspended in Buffer A and incubated with the anti- $\alpha$-taxilin antibody-coupled DYNABEADS M280 sheep anti-rabbit IgG (Invitrogen Corp.) in Buffer A containing $50 \mathrm{mM} \mathrm{NaCl}$ and $0.1 \%$ bovine serum albumin for $2 \mathrm{~h}$. After incubation, the magnetic beads were washed four times in Buffer A containing $50 \mathrm{mM} \mathrm{NaCl}$ and $0.1 \%$ bovine serum albumin and sequentially twice in Buffer A containing $50 \mathrm{mM} \mathrm{NaCl}$ according to the manufacturer's instructions. Proteins bound to the magnetic beads were eluted with Laemmli's sample buffer.

\section{Extraction of $\alpha$-taxilin from the membrane fraction}

All the following procedures were performed at $4{ }^{\circ} \mathrm{C}$. The membrane fraction of Hela cells prepared as described above was incubated for $1 \mathrm{~h}$ in $0.1 \mathrm{M} \mathrm{Na}_{2} \mathrm{CO}_{3}$ at $\mathrm{pH} 11.5$ or Buffer A containing the indicated concentrations of $\mathrm{NaCl}$, followed by centrifugation at $100,000 \times g$ for $1 \mathrm{~h}$. After centrifugation, the supernatant was collected. The surface of the resulting pellet was carefully washed once in the respective extract buffers, and subsequently suspended in comparable volume of Laemmli's sample buffer.

\section{Microtubule-binding assay}

To prepare polymerized tubulin, bovine brain tubulin (final concentration of $2.5 \mathrm{mg} / \mathrm{ml}$ ) was incubated in Buffer B (0.1 M PIPES/ $\mathrm{NaOH}$ at $\mathrm{pH} 6.8,1 \mathrm{mM}$ EGTA, and $1 \mathrm{mM} \mathrm{MgSO}_{4}$ ) containing 20 $\mu \mathrm{M}$ taxol and $2.5 \mathrm{mM} \mathrm{GTP}$ for $30 \mathrm{~min}$ at $37^{\circ} \mathrm{C}$. After incubation, the sample was centrifuged at $30,000 \times \mathrm{g}$ for $30 \mathrm{~min}$ at $20^{\circ} \mathrm{C}$. The resulting pellet was carefully washed once in Buffer B, subsequently suspended in the same buffer, and used as taxol-polymerized tubulin. To prepare $\mathrm{His}_{6}-\alpha$-taxilin- and GST-coupled magnetic beads, various amounts of each protein were incubated with the anti- $\alpha$ taxilin and anti-GST antibodies, respectively, coupled DYNABEADS M-280 sheep anti-rabbit IgG. In one set of experiments, $10 \mu \mathrm{g}$ of taxol-polymerized tubulin was incubated with the magnetic beads in $50 \mu \mathrm{l}$ of Buffer B containing $20 \mu \mathrm{M}$ taxol, $0.1 \%$ bovine serum albumin, and $50 \mathrm{mM} \mathrm{NaCl}$ for $30 \mathrm{~min}$ at $37^{\circ} \mathrm{C}$. After incubation, the magnetic beads were washed three times in Buffer B containing $2 \mu \mathrm{M}$ taxol, $0.1 \%$ bovine serum albumin, and $50 \mathrm{mM} \mathrm{NaCl}$, and sequentially once in Buffer B containing $2 \mu \mathrm{M}$ taxol and 50 $\mathrm{mM} \mathrm{NaCl}$ at $30^{\circ} \mathrm{C}$. In another set of experiments, $10 \mu \mathrm{g}$ of tubulin dimer was incubated with the magnetic beads at $4{ }^{\circ} \mathrm{C}$ in the same buffer conditions as described above, except for the absence of taxol. After incubation, the magnetic beads were washed at $4{ }^{\circ} \mathrm{C}$ according to the same procedures as described above, except for the absence of taxol. Proteins bound to the magnetic beads were eluted with Laemmli's sample buffer.

\section{Western blotting}

Protein concentrations were determined with bovine serum albumin as a reference protein as described (Bradford, 1976). SDSpolyacrylamide gel electrophoresis (PAGE) was performed as described (Laemmli, 1970). Western blotting was performed using the ECL-Plus immunoblotting detection system (GE Healthcare UK Ltd., Amersham Place, England) according to the manufacturer's instructions.

\section{Results}

\section{Association of $\alpha$-taxilin with intracellular components as a peripheral membrane protein}

To understand the cellular function of $\alpha$-taxilin, it is essential to determine it's intracellular distribution. First, to verify the specificity of the anti- $\alpha$-taxilin antibody used here, Hela cells, in which $\alpha$-taxilin was depleted by introducing siRNA oligo duplex against $\alpha$-taxilin, were stained with the anti- $\alpha$-taxilin antibody. The staining obtained by use of this antibody was disappeared in the cells where $\alpha$-taxilin was depleted (Fig. 1A and B), indicating that the anti- $\alpha$-taxilin antibody specifically stains endogenous $\alpha$-taxilin in Hela cells. When Hela cells were stained with the anti- $\alpha$-taxilin antibody, $\alpha$-taxilin was stained throughout the cytoplasm and often exhibited a tubulo-vesicular pattern, raising the possibility that $\alpha$-taxilin is associated with cytoskeletal components, especially microtubules. Then, we performed double-label immunofluorescence of $\alpha$-taxilin and $\alpha$-tubulin in Hela cells. The staining pattern of $\alpha$-taxilin almost overlapped with that of $\alpha$-tubulin (Fig. 1C and D). The result suggests that the microtubule cytoskeleton is involved in the intracellular distribution of $\alpha$-taxilin. Next, we performed double-label immunofluorescence of $\alpha$-taxilin and GM130, KDEL protein, or calnexin. GM130, and KDEL protein and calnexin are marker proteins of the Golgi apparatus and endoplasmic reticulum (ER), respectively, both of which are associated with the microtubule cytoskeleton. The staining pattern of $\alpha$-taxilin significantly overlapped with that of KDEL protein (Fig. 1F) but not with that of GM130 or calnexin (Fig. 1E and G). The depletion of $\alpha$-taxilin did not significantly affect the staining pattern of any marker protein described above (data not shown). Together, these results suggest that $\alpha$-taxilin is localized on some intracellular components associated with the microtubule cytoskeleton, particularly a part of the ER. Then, to reveal whether $\alpha$-taxilin is localized on intracellular components 
A
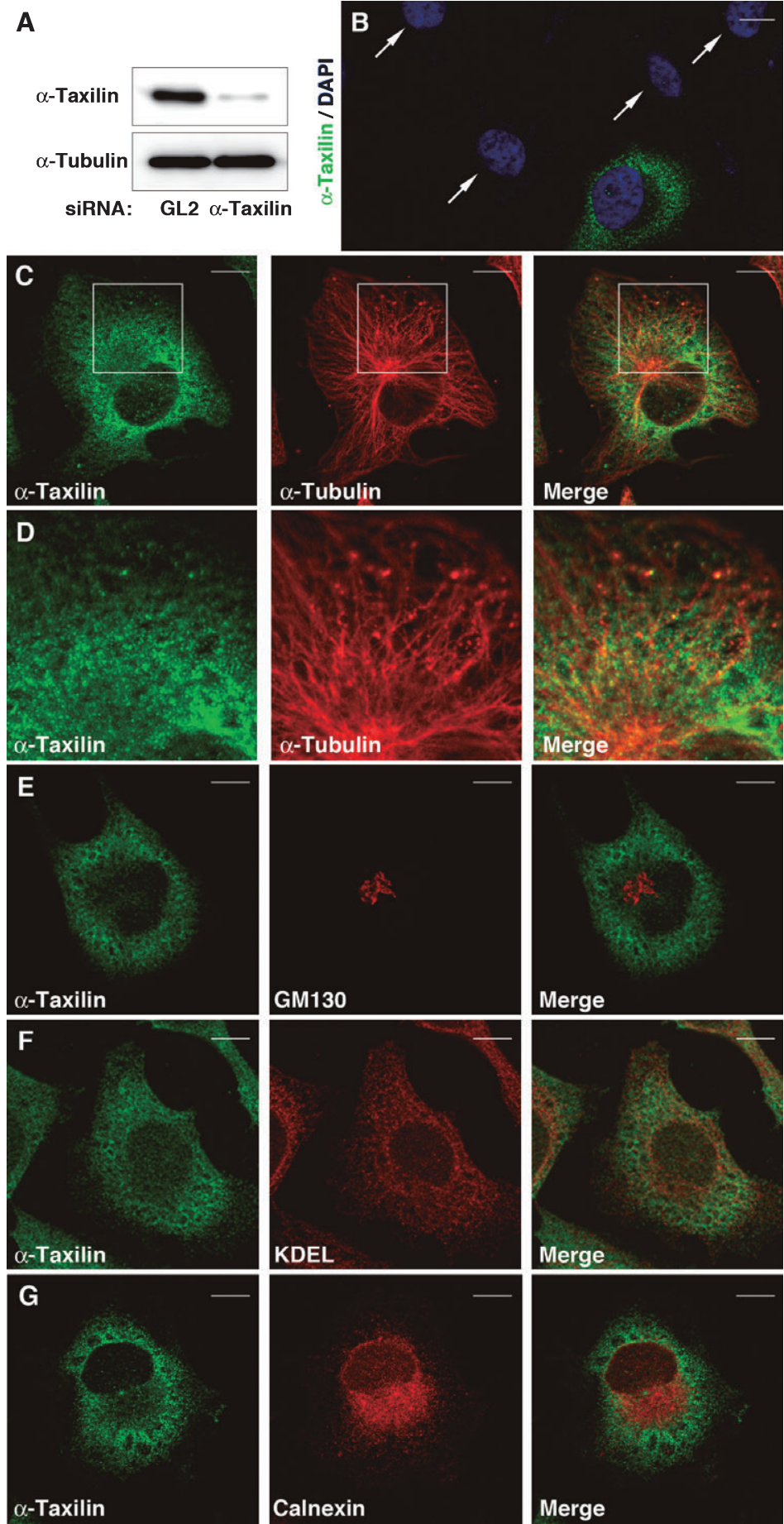

Fig. 1. Staining of $\alpha$-taxilin in a tubulo-vesicular pattern. (A and B) To deplete $\alpha$-taxilin in Hela cells, siRNA oligo duplex against $\alpha$-taxilin was introduced into the cells and further incubated for $72 \mathrm{~h}$. The control experiment was performed by use of siRNA oligo duplex against luciferase GL2. (A) A comparable amount of the post nuclear fraction of the treated cells was subjected to SDS-PAGE, followed by Western blotting with the anti- $\alpha$-taxilin and anti- $\alpha$-tubulin antibodies. (B) The cells treated with the siRNA oligo duplex against $\alpha$-taxilin were stained with the anti- $\alpha$-taxilin antibody and DAPI. Arrows indicate the cell where $\alpha$-taxilin was depleted. (C-G) Hela cells were double stained with the anti- $\alpha$-taxilin antibody and the anti- $\alpha$-tubulin $(\mathrm{C}$ and $\mathrm{D})$, the anti-GM130 (E), the anti-KDEL (F) or the anti-calnexin (G) antibody and analyzed by epifluorescence microscopy with an Apotome device. (D) Higher magnification images of the boxes of the respective images as shown in (C). The yellow color in merged images revealed the colocalization. Bar, $10 \mu \mathrm{m}$. 


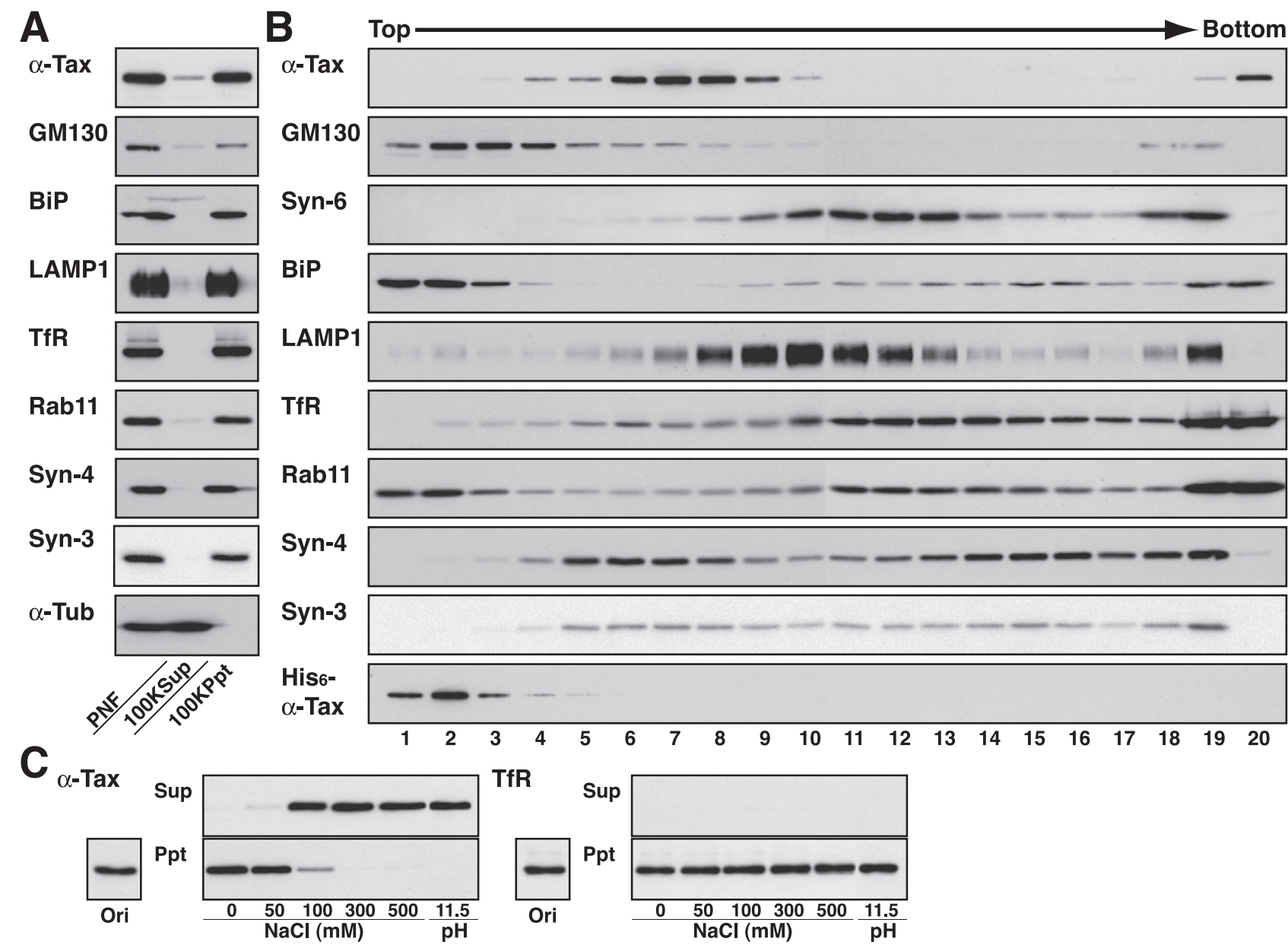

Fig. 2. Association of $\alpha$-taxilin with intracellular components as a peripheral membrane protein. (A) The post-nuclear fraction (PNF) of Hela cells was separated into the cytosol (100KSup) and membrane (100KPpt) fractions. A comparable amount of each fraction was subjected to SDS-PAGE, followed by Western blotting with the indicated antibodies. (B) In one set of experiments, the membrane fraction was further fractionated with OptiPrep. In another set of experiments, purified $\mathrm{His}_{6}-\alpha$-taxilin was fractionated with OptiPrep under the same conditions. An aliquot of each fraction was subjected to SDS-PAGE, followed by Western blotting with the indicated antibodies. The following proteins were used for cytosol and organelle markers; $\alpha$-Tub (cytosol); GM130 (Golgi), Syn-6 (TGN), BiP (ER), LAMP1 (lysosomes), TfR and Rab11 (recycling endosomes), and Syn-3 and -4 (plasma membrane). (C) The membrane fraction was incubated in the indicated extract buffers. After incubation, the sample was centrifuged at $100,000 \times \mathrm{g}$ for $1 \mathrm{~h}$ to separate to the soluble (Sup) and pellet (Ppt) fractions. A comparable amount of each fraction was subjected to SDS-PAGE followed by Western blotting with the anti- $\alpha$-taxilin and anti-TfR antibodies.

or not, we performed subcellular fractionation. $\alpha$-Taxilin was abundantly present in the membrane fraction and to a lesser extent in the cytosol fraction (Fig. 2A). When the membrane fraction was further fractionated with OptiPrep, $\alpha$-taxilin appeared mainly in Fractions 4-10 and to a lesser extent in the bottom fraction (Fig. 2B). The fractions including $\alpha$-taxilin were substantially separated from Golgi vesicles (GM130; Fractions 1-5), trans-Golgi network (TGN)-derived membrane vesicles (Syntaxin-6; Fractions 9-14), ER-derived membrane vesicles (BiP; Fractions 9-17), lysosomes (LAMP1; Fractions 8-13), recycling endosomes (TfR and Rab11; Fractions 10-18), and plasma membrane (Syntaxin-3 and -4; Fractions 12-19). Although the reason why both of $\mathrm{BiP}$ and Rab11 additionally appeared in Fractions 1-3 remains unclear, it is possible that these proteins were dissociated from the respective localized organelles during the experimental procedures. Under the same conditions, purified His $_{6}$-tagged $\alpha$-taxilin appeared in Fractions 1-3 (Fig. 2B). These results indicate that $\alpha$-taxilin is abundantly localized on the intracellular components which are at least distinct from the organelles investigated here.

A structural analysis of $\alpha$-taxilin has revealed that $\alpha$ taxilin has no transmembrane segment (Nogami et al., 2003a). Therefore, there are at least the following two possibilities concerning a mode of the association of $\alpha$-taxilin 
with the intracellular components; one is that $\alpha$-taxilin is associated with the intracellular components through its interaction with a transmembrane protein. The other is that $\alpha$-taxilin undergoes post-translational lipid-modifications and is associated with the intracellular components through lipid-phospholipid interactions. To resolve this issue, the membrane fraction of Hela cells was treated with various concentrations of $\mathrm{NaCl}$ and subsequently subjected to centrifugation to separate the soluble and insoluble fractions. Each fraction was then subjected to SDS-PAGE, followed by Western blotting with the anti- $\alpha$-taxilin and anti-TfR antibodies. The treatment of the membrane fraction with $0.5 \mathrm{M} \mathrm{NaCl}$ induced an absolute extraction of $\alpha$-taxilin from the membrane fraction (Fig. 2C). The extraction of $\alpha$-taxilin was dependent on the concentrations of $\mathrm{NaCl}$ (Fig. 2C). In contrast, TfR, a transmembrane protein, was detected in the insoluble fraction but not in the soluble fraction irrespective of the concentrations of $\mathrm{NaCl}$. Moreover, the treatment of the membrane fraction with $0.1 \mathrm{M} \mathrm{Na}_{2} \mathrm{CO}_{3}$ at $\mathrm{pH} 11.5$ also induced an absolute extraction of $\alpha$-taxilin from the membrane fraction, whereas the same treatment did not induce extraction of TfR from the membrane fraction (Fig. 2C). These results indicate that $\alpha$-taxilin is a peripheral membrane protein, raising a question of which molecule is required for the association of $\alpha$-taxilin with the intracellular components. It has been revealed that in normal rat kidney cells, syntaxin- 3 and -4 are mainly localized on the plasma membrane but to some extents on vesicular organelles (Band et al., 2002, Band and Kuismanen, 2005). Consistently, when the membrane fraction of Hela cells was fractionated with OptiPrep, both of syntaxin-3 and -4 appeared as two peaks (Fractions 5-8 and Fractions 12-19). The former peak partially overlapped with that of $\alpha$-taxilin. Taken together with our previous report that among the syntaxin family members, syntaxin- 3 and -4 more favorably interacts with $\alpha$-taxilin, the results raise the possibility that $\alpha$-taxilin is associated with the intracellular components through its interaction with syntaxin-3 and/or -4 . Then, to address the issue, two sets of experiments were performed. First, the $\alpha$-taxilin-containing intracellular components were immunoabsorbed from the membrane fraction of Hela cells by use of the anti- $\alpha$-taxilin antibody-coupled magnetic beads. When the $\alpha$-taxilin-containing intracellular components were almost immunoabsorbed, neither syntaxin-3 nor -4 was co-immunoabsorbed (Fig. 3A). Second, syntaxin-3 or -4 was depleted in Hela cells by introducing siRNA oligo duplex against the respective molecules. When the treated cells were roughly fractionated to the cytosol and membrane fractions, depletion of either syntaxin hardly affected the subcellular distribution of $\alpha$-taxilin (Fig. 3B). Moreover, immunofluorescence analysis showed that the depletion of syntaxin-4 did not affect the intracellular distribution of $\alpha$-taxilin and that $\alpha$-taxilin was hardly co-localized with syntaxin-4 in the cells, where syntaxin- 4 was not depleted (Fig. 3C). Finally, syntaxin-18, a syntaxin member resided on the ER, was depleted in Hela cells by introducing siRNA oligo duplex against syntaxin-18 as previously described (Iinuma et al., 2009). When the treated cells were roughly fractionated to the cytosol and membrane fractions, depletion of syntaxin-18 hardly affected the subcellular distribution of $\alpha$-taxilin (Fig. 3D). Taken together, these results indicate that $\alpha$-taxilin is associated with the intracellular components through its interaction with an unknown molecule other than syntaxin-3, -4 or -18 .

\section{Microtubule-dependent distribution of $\alpha$-taxilin-containing intracellular components}

In Hela cells, the staining pattern of $\alpha$-taxilin often exhibited a tubulo-vesicular pattern and significantly overlapped with that of KDEL protein as shown in Fig. 1, raising the possibility that $\alpha$-taxilin is associated with a part of the ER. However, this is inconsistent with the results obtained by biochemical analysis as shown in Fig. 2. Then, to more closely characterize the $\alpha$-taxilin-containing intracellular components, the effects of reversible microtubule depolymerization with nocodazole on the distribution of the $\alpha$ taxilin-containing intracellular components were investigated. Depolymerization of microtubules with nocodazole disrupted a typical microtubule structure (Fig. 4A) and, moreover, induced dispersions of GM130 and calnexin throughout the cytoplasm (Fig. 5A and D). Concomitantly, the tubulo-vesiclular pattern of $\alpha$-taxilin was almost disappeared, although $\alpha$-taxilin was still partially localized at the tubulo-vesicular structure around the perinuclear region (Fig. 4A). The staining for $\alpha$-taxilin was diffusely detected throughout the cytoplasm and to a more extent at the peripheral region (Fig. 4A). Although a physiological significance of the accumulation of $\alpha$-taxilin at the peripheral region remains unclear, it is possible that since depolymerization of tubulin was regionally incomplete, $\alpha$-taxilin was localized at the tubulo-vesicular structure in the region where $\alpha$ tubulin was localized at the tubulo-vesicular structure. Next, nocodazole was washed out of the cells and the behaviors of microtubules, GM130, calnexin, and $\alpha$-taxilin were observed. Many microtubule fragments were observed after $1 \mathrm{~h}$ recovery (Fig. 4B). There was approximately the normal number of microtubule fragments associated with the microtubule organizing center after $2 \mathrm{~h}$ recovery (Fig. 4C). Although the recovery of calnexin was more slowly than those of microtubules, GM130, and $\alpha$-taxilin, the recoveries of all proteins were complete in most cells after $2 \mathrm{~h}$ recovery. GM130 was moved to a juxtanuclear position after $1 \mathrm{~h}$ recovery (Fig. 5B). After $2 \mathrm{~h}$ recovery, almost all of GM130 was concentrated at the perinuclear region, showing the perinuclear structural reorganization of the Golgi apparatus (Fig. 5C). After $1 \mathrm{~h}$ recovery, the recovery of $\alpha$-taxilin was almost complete in most cells and its tubulovesicular pattern was observed throughout the cytoplasm (Fig. 5B). On the other hand, after $1 \mathrm{~h}$ recovery, calnexin 


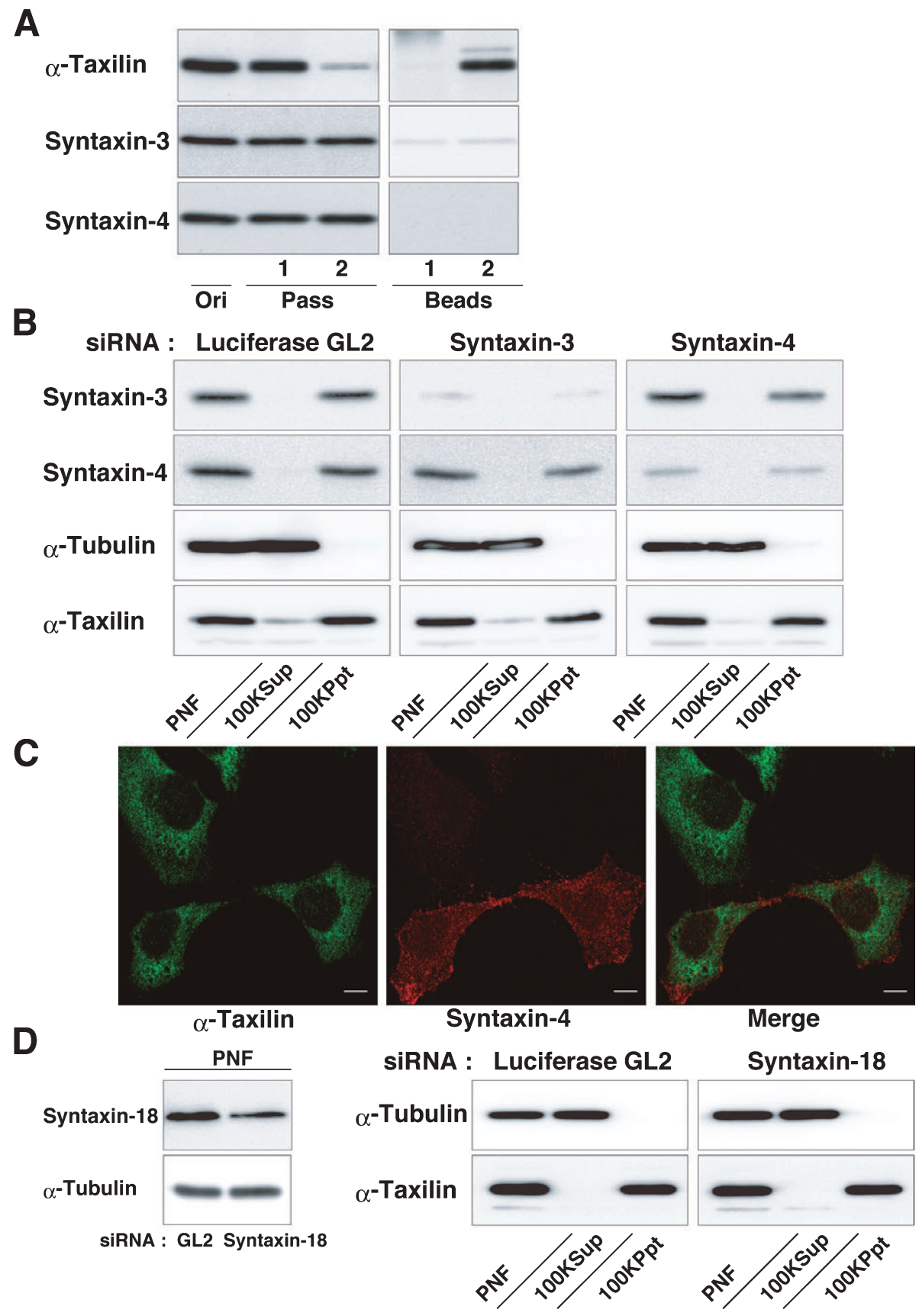

Fig. 3. Intracellular distribution of $\alpha$-taxilin in a syntaxin-independent manner. (A) To immunoabsorb $\alpha$-taxilin-containing intracellular components from the membrane fraction, the membrane fraction of Hela cells was incubated with the control rabbit Ig coupled to the magnetic beads (1) or the anti- $\alpha$-taxilin antibody coupled to the magnetic beads (2). A comparable amount of each sample was subjected to SDS-PAGE, followed by Western blotting with the anti$\alpha$-taxilin, anti-syntaxin-3, and anti-syntaxin-4 antibodies. (B, C, and D) To deplete syntaxin-3, -4 , or -18 in Hela cells, siRNA oligo duplex against the respective molecules was introduced into the cells and further incubated for $72 \mathrm{~h}$. The control experiment was performed by use of siRNA oligo duplex against luciferase GL2. (B and D) In one set of experiments, the cells were fractionated into three fractions including the post-nuclear (PNF), cytosol (100KSup), and membrane (100KPpt). A comparable amount of each fraction was subjected to SDS-PAGE, followed by Western blotting with the indicated antibodies. (C) In another set of experiments, the cells were double stained with the anti- $\alpha$-taxilin and anti-syntaxin- 4 antibodies and analyzed by epifluorescence microscopy with an Apotome. The yellow color in merged image revealed the colocalization. Bar, $10 \mu \mathrm{m}$. 


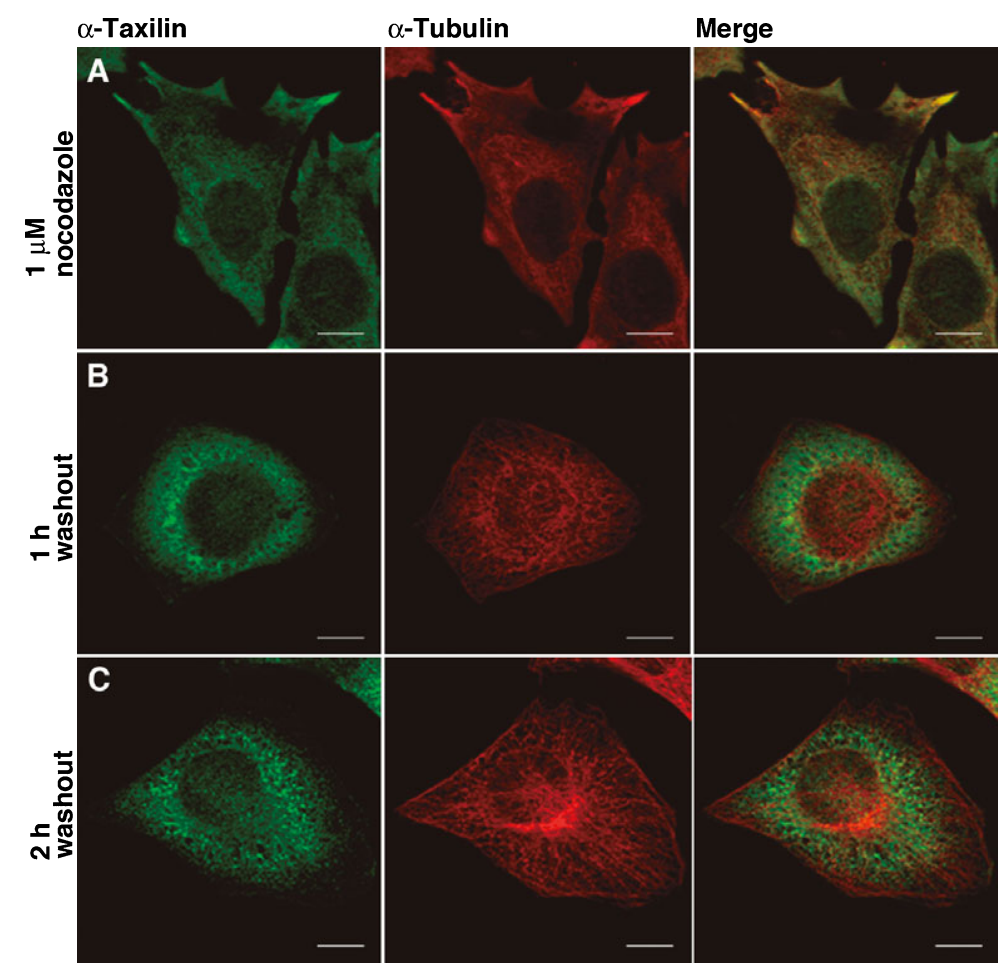

Fig. 4. Reversible effect of nocodazole on the intracellular distribution of $\alpha$-taxilin. Hela cells were treated with nocodazole (A). The cells treated with nocodazole were further incubated in the absence of nocozazole for $1 \mathrm{~h}(\mathrm{~B})$ and $2 \mathrm{~h}(\mathrm{C})$ after treatment with nocodazole. The cells were double stained with the anti- $\alpha$-taxilin and anti- $\alpha$-tubulin antibodies and analyzed by epifluorescence microscopy with an Apotome device. The yellow color in merged images revealed the colocalization. Bar, $10 \mu \mathrm{m}$.

was localized around the perinuclear region but still to a significant extent at the peripheral region (Fig. 5E). At $2 \mathrm{~h}$ recovery, calnexin was abundantly localized around the perinuclear region (Fig. 5F), being similar to the subcellular distribution of calnexin as shown in Fig. 1G. It is notable that after nocodazole was washed out, the behavior of calnexin was not completely overlapped with that of $\alpha$-taxilin. Particularly, at $2 \mathrm{~h}$ recovery, the tubulo-vesicular pattern of $\alpha$-taxilin was hardly detected in the perinuclear region where calnexin was abundantly detected. Biochemical analysis as shown in Fig. 2 indicates that $\alpha$-taxilin is localized on intracellular components under the experimental conditions, where tubulin is depolymerized. Therefore, it is likely that the behavior of $\alpha$-taxilin affected by the reversible microtubule depolymerization with nocodazole represents that of the $\alpha$-taxilin-containing intracellular components. Together, these results indicate that the distribution of the $\alpha$-taxilin-containing intracellular components is dependent on the microtubule cytoskeleton and that $\alpha$-taxilin is localized on some intracellular components other than the ER. However, from our present data, the possibility that the $\alpha$ taxilin-containing intracellular components have a close relation with a part of the ER cannot yet be excluded.

\section{Direct interaction of $\alpha$-taxilin with polymerized tubulin}

It is often that a microtubule-binding protein resided on an intracellular component is implicated as a linker protein in the association of the intracellular component with the microtubule cytoskeleton. According to a structural analysis of $\alpha$-taxilin, $\alpha$-taxilin does not posses any characteristic microtubule-binding domain revealed so far. However, the possibility that $\alpha$-taxilin functions as a linker protein in the association of the $\alpha$-taxilin-containing intracellular components with the microtubule cytoskeleton cannot be excluded. Then, we examined whether $\alpha$-taxilin directly interacts with tubulin in vitro. When tubulin dimer was incubated with $\mathrm{His}_{6}-\alpha$-taxilin coupled to the magnetic beads, tubulin dimer did not bind to $\mathrm{His}_{6}-\alpha$-taxilin (Fig. 6A). However, when taxol-polymerized tubulin was incubated with $\mathrm{His}_{6}-\alpha$-taxilin coupled to the magnetic beads, taxol-polymerized tubulin surprisingly bound to $\mathrm{His}_{6}-\alpha-$ taxilin (Fig. 6B). The interaction of taxol-polymerized tubulin with $\mathrm{His}_{6}-\alpha$-taxilin was dependent on the amounts of $\mathrm{His}_{6}$ - $\alpha$-taxilin coupled to the magnetic beads and inhibited by $0.5 \mathrm{M} \mathrm{NaCl}$ (Fig. 6B). Under the same conditions, taxol-polymerized tubulin did not bind to GST coupled to the magnetic beads (Fig. 6B). The results indicate that $\alpha$ taxilin directly interacts with polymerized tubulin but not 


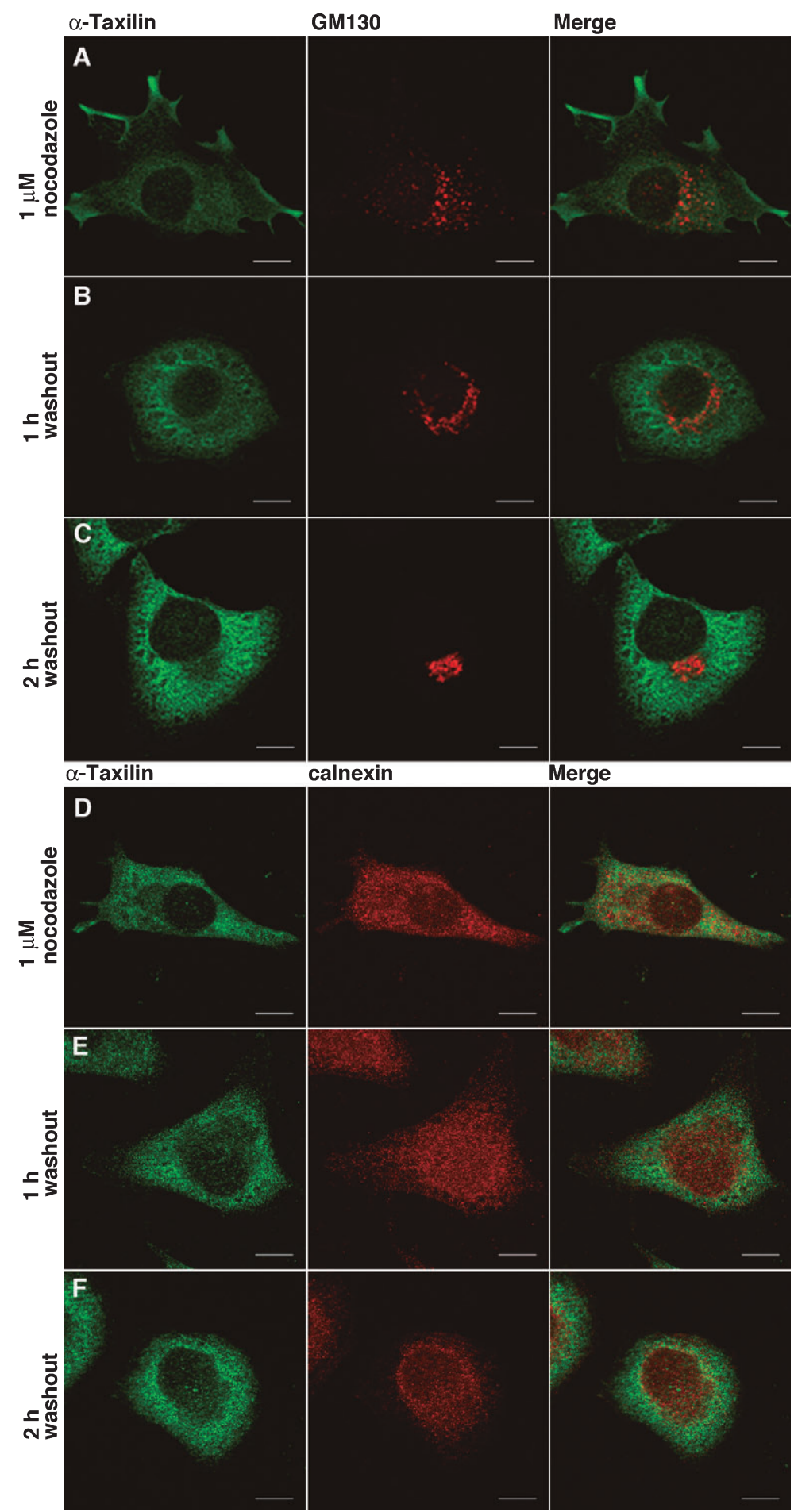

Fig. 5. Association of $\alpha$-taxilin with intracellular components other than the Golgi apparatus and ER. Hela cells were treated with nocodazole (A and D). The cells treated with nocodazole were further incubated in the absence of nocozazole for $1 \mathrm{~h}(\mathrm{~B}$ and $\mathrm{E})$ and $2 \mathrm{~h}(\mathrm{C}$ and $\mathrm{F})$ after treatment with nocodazole. The cells were double stained with the anti- $\alpha$-taxilin antibody and the anti-GM130 (A, B, and C) or the anti-calnexin (D, E, and F) antibody and analyzed by epifluorescence microscopy with an Apotome device. The yellow color in merged images revealed the colocalization. Bar, $10 \mu \mathrm{m}$. 


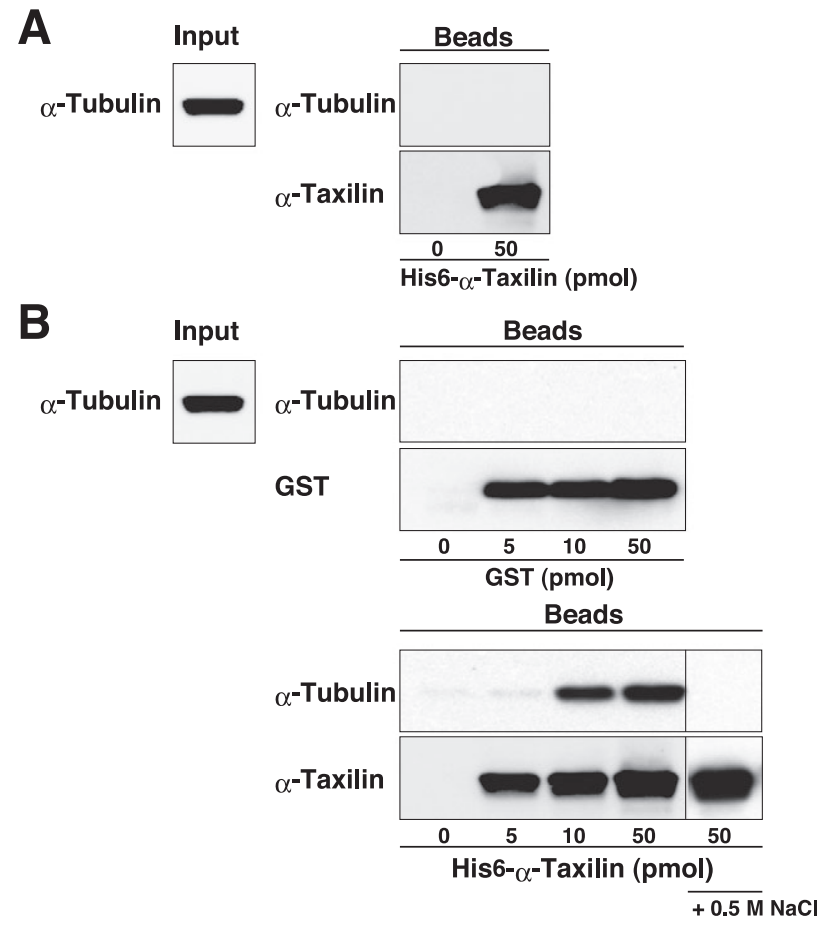

Fig. 6. Direct interaction of $\alpha$-taxilin with polymerized tubulin. Tubulin dimer (A) or taxol-polymerized tubulin (B) was incubated with the indicated amounts of $\mathrm{His}_{6}-\alpha$-taxilin or GST coupled to the magnetic beads in the presence or absence of $0.5 \mathrm{M} \mathrm{NaCl}^{\text {. His }}{ }_{6}-\alpha$-taxilin, GST, and tubulin bound to the beads were detected by Western blotting with the anti- $\alpha$ taxilin, anti-GST, and anti- $\alpha$-tubulin antibodies, respectively.

with tubulin dimer in vitro and suggest that $\alpha$-taxilin functions as a linker protein in the association of the $\alpha$ taxilin-containing intracellular components with the microtubule cytoskeleton.

\section{Brefeldin A-insensitive and NEM-sensitive intracellular distribution of $\alpha$-taxilin}

Finally, we examined the effects of two intracellular vesicle traffic-perturbing drugs on the intracellular distribution of $\alpha$-taxilin. First, Hela cells were treated with brefeldin A, which is a fungal metabolite to inhibit intracellular vesicle traffic by selective disruption of the Golgi apparatus (Fujiwara et al., 1988; Helms and Rothman, 1992). The treatment with brefeldin A induced dispersion of GM130 throughout the cytoplasm (Fig. 7A). However, under the same conditions, $\alpha$-taxilin exhibited a staining pattern similar to that seen in the cells under normal culture conditions and was not overlapped with GM130 (Fig. 7A). The result indicates that $\alpha$-taxilin is at least localized on brefeldin Ainsensitive intracellular components. Next, we investigated whether the inhibition of the SNARE complex disassembly with NEM affects the intracellular distribution of $\alpha$-taxilin or not. NEM is a sulfhydryl alkylating reagent to inactivate
NSF (Block et al., 1988). It has been reported that NEM inhibits intracellular vesicle traffic from the trans-Golgi network to the plasma membrane (Band et al., 2001). The treatment with NEM induced dispersion of $\alpha$-taxilin throughout the cytoplasm and nucleus (Fig. 7B-D, Fig. 8B and D). In the peripheral region, a part of $\alpha$-taxilin exhibited a tubulo-vesicular pattern similar to that as shown in the cells under the normal culture conditions. Surprisingly, $\alpha-$ taxilin was accumulated in the nucleus in a part of the NEM-treated cells. Under the same conditions, no significant change was observed in the staining pattern of GM130 or syntaxin-4 (Fig. 8A-D). GM-130 was localized at the perinuclear region in the cells without NEM-treatment and the perinuclear distribution was not significantly affected by NEM-treatment. Syntaxin-4 was diffusely localized in the cells without NEM-treatment and the diffuse subcellular distribution was not significantly affected by NEM-treatment. However, significant changes were observed in the staining pattern of $\alpha$-tubulin and KDEL protein (Fig. 7B-D). The staining pattern of $\alpha$-tubulin was morphologically distinct from a typical microtubule structure but significantly overlapped with that of $\alpha$-taxilin in the cytoplasm (Fig. 7B and C). A typical tubulo-vesicular pattern of KDEL protein was disappeared, and a diffuse dot-like staining for KDEL protein was detected around the perinuclear region but not overlapped with that for $\alpha$-taxilin (Fig. 7D). Either $\alpha$ tubulin or KDEL protein was not localized in the nucleus (Fig. 7B-D). To verify the nuclear localization of $\alpha$-taxilin in the NEM-treated cells, we investigated whether the treatment with NEM induces nuclear localization of exogenously expressed $\alpha$-taxilin or not. When EGFP- and myc$\alpha$-taxilins were exogenously expressed in Hela cells, these proteins were distributed in the cytoplasm but not in the nucleus (Fig. 9A and C). However, EGFP- and myc- $\alpha-$ taxilins were accumulated in the nucleus in the NEMtreated cells (Fig. 9B and D). These results indicate that NEM treatment affects the intracellular distribution of $\alpha$ taxilin and suggest that NEM treatment does not affect the association of $\alpha$-taxilin with the altered microtubule cytoskeleton.

\section{Discussion}

Using immunofluorescence and subcellular fractionation analyses, we have shown here that $\alpha$-taxilin is abundantly present on the membrane fraction and to a lesser extent on the cytosol fraction in Hela cells and that $\alpha$-taxilin is associated with intracellular components as a peripheral membrane protein. The $\alpha$-taxilin-containing intracellular components were distinct from the ER, Golgi apparatus, endosomes, or lysosomes. Recently, it has been revealed that syntabulin, a syntaxin-1-binding protein, is localized on syntaxin-1-cargo vesicles through its interaction with syntaxin-1 and subsequently mediates the transport of 

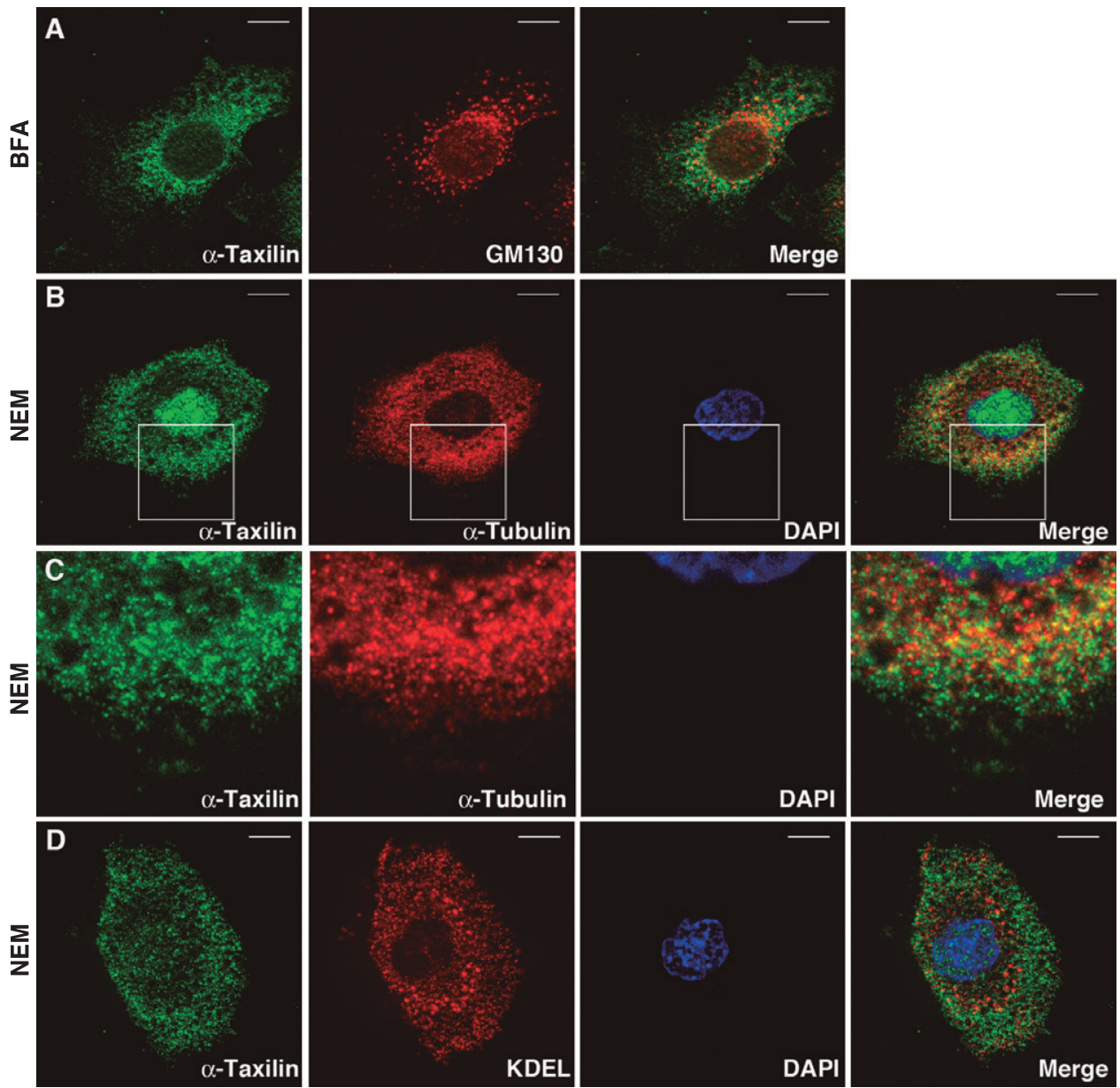

Fig. 7. Disturbance of the intracellular distribution of $\alpha$-taxilin by treatment with NEM but not with brefeldin A. Hela cells were treated with brefeldin A (A). Hela cells were treated with NEM (B-D). The cells were double stained with the anti- $\alpha$-taxilin antibody and the anti-GM130 (A), the anti- $\alpha$-tubulin (B and C), or the anti-KDEL (D) antibody. The NEM-treated cells were furthermore stained with DAPI (B-D). The stained cells were analyzed by epifluorescence microscopy with an Apotome device. (C) Higher magnification images of the boxes of the respective images as shown in (B). The yellow color in merged images revealed the colocalization. Bar, $10 \mu \mathrm{m}$.

syntaxin-1 to neuronal processes (Cai et al., 2007b; Su et al., 2004). Therefore, it is possible that $\alpha$-taxilin is also associated with syntaxin-cargo vesicles. We have previously revealed that $\alpha$-taxilin preferentially binds to syntaxin- 4 , and moderately to syntaxin- 1 and -3 but not to syntaxin- 7 , a syntaxin member resided on late endosome and lysosome, or syntaxin-8, a syntaxin member resided on early endosome (Nogami et al., 2003a). It has been revealed that syntaxin-3 and -4 but not syntaxin- 1 are abundantly present in nonneuronal tissues including Hela cells and that in normal rat kidney cells, syntaxin- 3 and -4 are localized on the TGN and rab11-positive endosomal membranes, respectively, in addition to the plasma membrane (Band et al., 2002, Band and Kuismanen, 2005). However, the present results obtained by RNAi and immunoabsorption analyses indicate that $\alpha$-taxilin is not localized on the vesicles containing syntaxin-3 or -4 . Moreover, nocodazole treatment markedly changed the staining pattern of $\alpha$-taxilin but not of syntaxin4 (data not shown). Taken together, it is likely that $\alpha$-taxilin is localized on some intracellular components other than syntaxin-cargo vesicles. On the basis of the present results, we propose the following mode of action of $\alpha$-taxilin in intracellular vesicle traffic. The extraordinarily long coiledcoil region of $\alpha$-taxilin is homologous to that of Uso1, a yeast tethering component (Nogami et al., 2003a). We have revealed that $\alpha$-taxilin interacts with syntaxin- 1 before the formation of the trans-SNARE complex (Nogami et al., $2003 \mathrm{~b}$ ). Assuming that $\alpha$-taxilin localized on intracellular components interacts with syntaxin-3 and -4 localized on the plasma membrane, it is possible that the $\alpha$-taxilincontaining intracellular components are tethered to the plasma membrane through the interaction of $\alpha$-taxilin with syntaxin-3 or -4 . In the future, it is important for understanding the role of $\alpha$-taxilin in intracellular vesicle traffic 

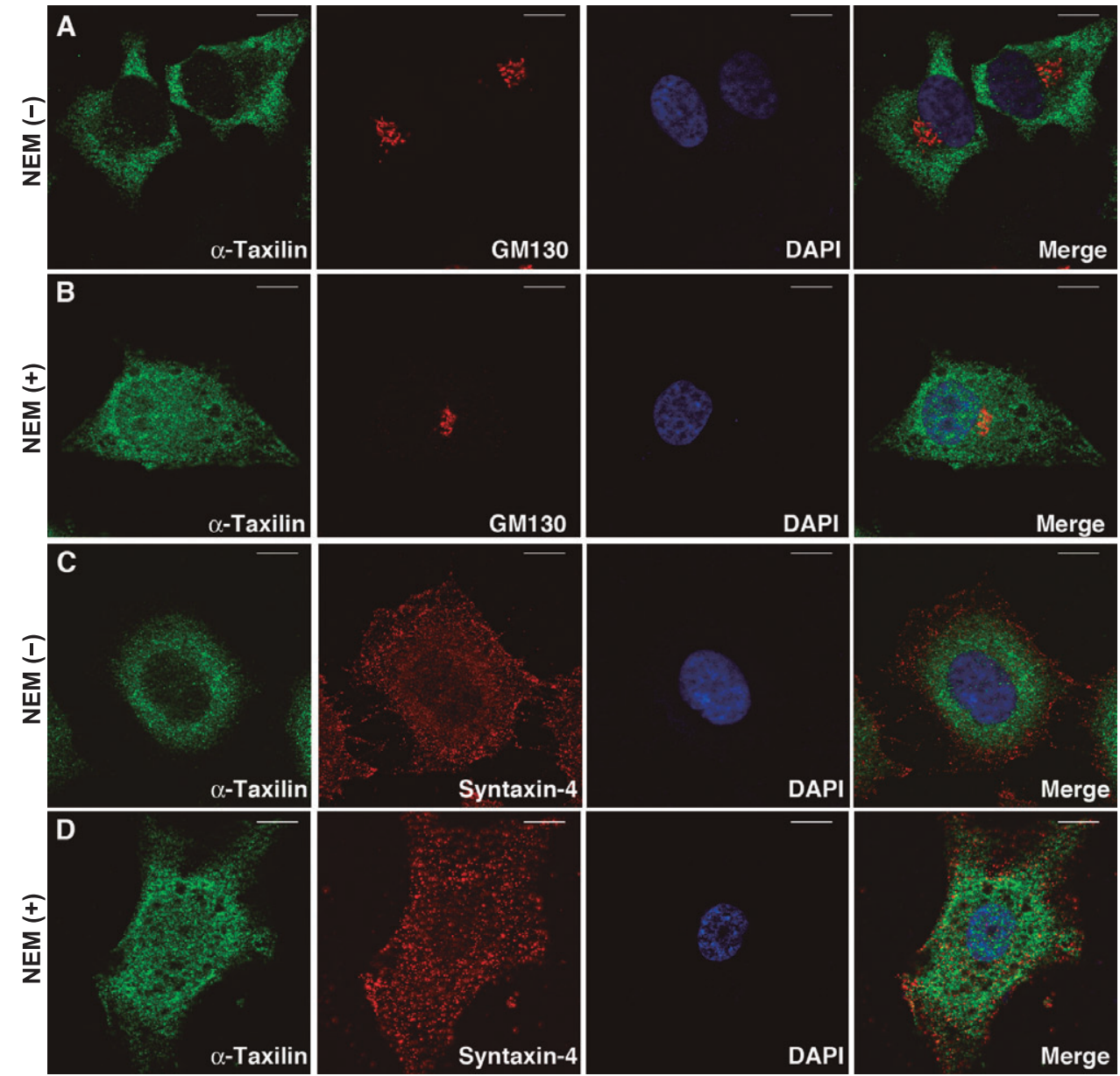

Fig. 8. Intracellular distribution of $\alpha$-taxilin compared with those of GM130 and syntaxin-4 in NEM-treated cells. Hela cells were treated with (B and D) or without (A and C) NEM. The cells were triple stained with the anti- $\alpha$-taxilin antibody, DAPI, and the anti-GM130 (A and B) or the anti-syntaxin-4 (C and D) antibody. The stained cells were analyzed by epifluorescence microscopy with an Apotome device. The yellow color in merged images revealed the colocalization. Bar, $10 \mu \mathrm{m}$.

to identify the $\alpha$-taxilin-containing intracellular components and clarify the mechanism by which $\alpha$-taxilin is associated with the intracellular components.

Since the $\alpha$-taxilin-containing intracellular components were distributed in Hela cells in a microtubule-dependent manner, it is an important issue to reveal whether $\alpha$-taxilin is involved in the association of the $\alpha$-taxilin-containing intracellular components with the microtubule cytoskeleton. It has been revealed that CLIP-170 and MAP2, both of which are microtubule-binding proteins, link endocytic vesicles and chromaffin granules, respectively, to the microtubule cytoskeleton (Pierre et al., 1992; Severin et al., 1991). Then, we have performed an in vitro binding assay using purified samples. Although $\alpha$-taxilin does not contain any characteristic microtubule-binding sites including CAP-Gly domains which are present in CLIP-170 and tandem repeats which are present in Tau, MAP2, and MAP4 proteins (Brandt and Lee, 1993; Perez et al., 1999;
Steinmetz and Akhmanova, 2008), $\alpha$-taxilin directly bound to taxol-polymerized tubulin but not to tubulin dimer. The result implies that $\alpha$-taxilin plays a role as a linker protein between the $\alpha$-taxilin-containing intracellular components and the microtubule cytoskeleton and, moreover, raises the possibility that the interaction of $\alpha$-taxilin with the microtubule cytoskeleton mediates the intracellular traffic of the $\alpha$-taxilin-containing intracellular components. Moreover, accumulating evidence indicates that several of the molecules which have been initially identified as SNARE-related molecules bind to microtubule and modulate its dynamics (Honda et al., 2002; Wang et al., 2004). It is possible that $\alpha$-taxilin also modulate microtubule dynamics. In the future, it is necessary not only to reveal the mechanism which underlies the interaction of $\alpha$-taxilin with the microtubule cytoskeleton but also to examine effect of $\alpha$-taxilin on microtubule dynamics.

$\alpha$-Taxilin was present in the cytoplasm to a lesser extent. 

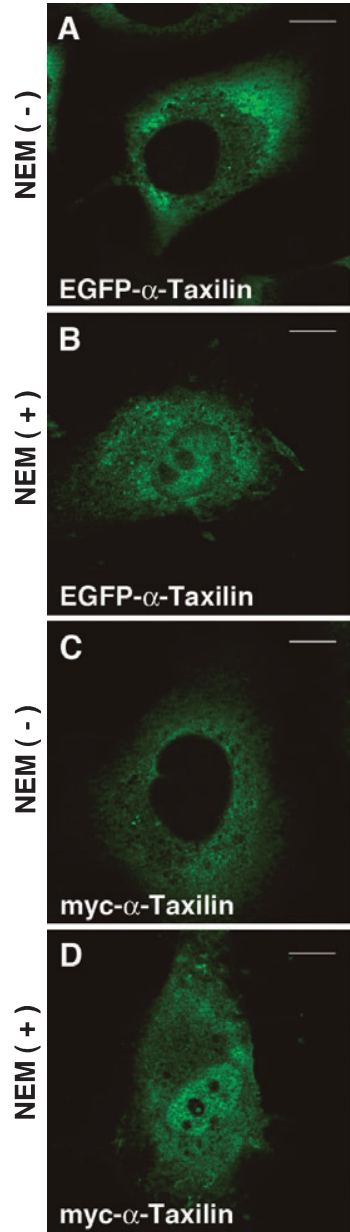
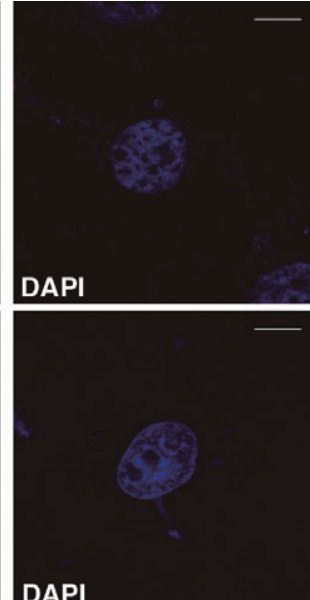

DAPI

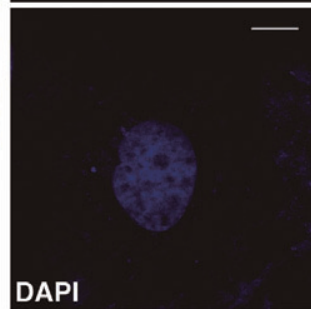

DAPI

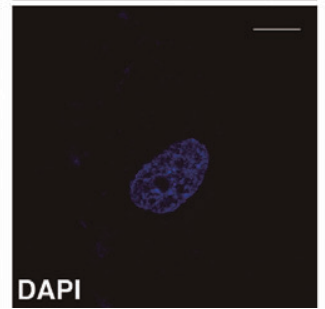

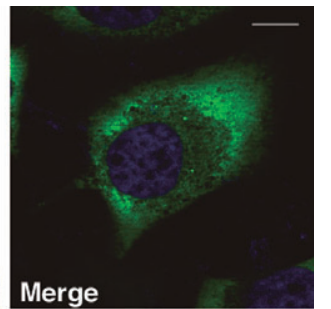

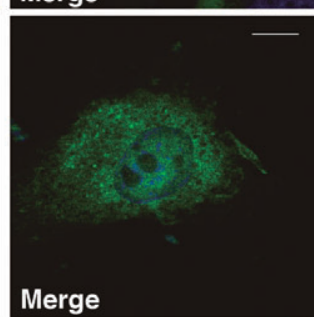

Merge
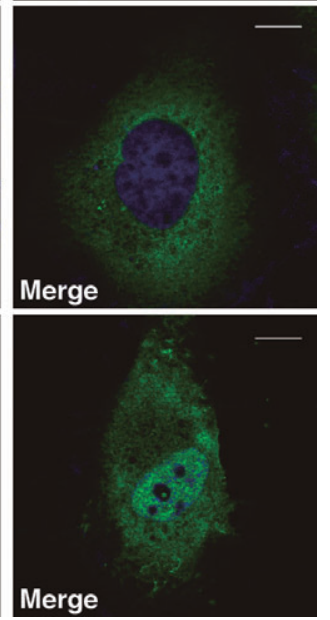

Fig. 9. Nuclear localization of exogenously expressed $\alpha$-taxilin by treatment with NEM. EGFP- and myc- $\alpha$-taxilins were exogenously expressed in Hela cells. The cells were treated with (B and D) or without NEM (A and C). The cells expressing EGFP- $\alpha$-taxilin were stained with DAPI (A and B). The cells expressing myc- $\alpha$-taxilin were double stained with the anti-myc antibody and DAPI (C and D). The stained cells were analyzed by epifluorescence microscopy with an Apotome device. Bar, $10 \mu \mathrm{m}$.

The staining pattern of $\alpha$-taxilin was more punctate than that of $\alpha$-tubulin and detected in the region spaced between the microtubule cytoskeleton. Now, although the difference between $\alpha$-taxilins present in the cytosol and membrane fractions remains unclear, we cannot yet exclude the possibility that $\alpha$-taxilin is involved in physiological processes other than intracellular vesicle traffic. We have recently found that the taxilin family interacts with nascent polypeptideassociated complex (NAC) composed of $\alpha$ and $\beta$ subunits $(\alpha-$ and $\beta \mathrm{NAC}$ ) and that the overexpression of the taxilin family inhibits the translocation of $\alpha \mathrm{NAC}$ from the cytosol to the nucleus (Yoshida et al., 2005). NAC is an abundant cytosolic protein and implicated in transferring growing nascent polypeptides to appropriate co-translationally acting factors through its interaction with the nascent chains on the ribosome (Rospert et al., 2002). Moreover, accumulating evidence suggests that $\alpha \mathrm{NAC}$ is translocated from the cytosol to the nucleus and subsequently regulate the transcrip- tional process. Therefore, it is possible that a small part of $\alpha$-taxilin is involved in the NAC-related functions.

The treatment of Hela cells with NEM induces changes of the intracellular distributions of $\alpha$-taxilin, $\alpha$-tubulin, and KDEL protein but not of GM130 or syntaxin-4. However, the altered staining pattern of $\alpha$-taxilin but not of KDEL protein in the cytoplasm was still overlapped with that of $\alpha$-tubulin, which was distinct from a typical microtubule structure. It has been revealed that NEM inhibits microtubule minus end assembly through the modification of tubulin (Phelps and Walker, 2000), raising the possibility that the altered microtubule structure caused by treatment with NEM indirectly induces the change of the distribution of $\alpha$-taxilin, probably $\alpha$-taxilin-containing intracellular components, in the cytoplasm. However, since several of the NEM-treated cells exhibited the accumulation of $\alpha$ taxilin but not of $\alpha$-tubulin in the nucleus, it is likely that $\alpha$-taxilin free of intracellular components is transported into 
the nucleus in a tubulin-independent manner. Therefore, it is possible that $\alpha$-taxilin and/or $\alpha$-taxilin-related molecules other than tubulin are directly modified by treatment with NEM, resulting in the nuclear localization of $\alpha$-taxilin. Since, in this study, we could not find any physiological signals inducing the nuclear localization of $\alpha$-taxilin, a physiological significance of the nuclear localization of $\alpha$-taxilin induced by NEM treatment remains unclear. However, after we had reported the interaction of the taxilin family with $\alpha$ - and $\beta$ NACs, Rene St-Arnaud and colleague have identified a protein identical to $\gamma$-taxilin in a yeast two-hybrid screen for proteins interacting with $\alpha \mathrm{NAC}$ and named it factor inhibiting activating transcription factor 4 (ATF4)-mediated transcription (FIAT) (Yu et al., 2005). They have found that in calvarial osteoblasts from primary cultures, $\gamma$-taxilin/FIAT localizes to the nucleus, interacts with ATF4, and blocks ATF4-mediated transcription of the osteocalcin gene ( $\mathrm{Yu}$ et al., 2005, 2006). Therefore, it is possible that under certain conditions, $\alpha$-taxilin is also translocated to the nucleus and subsequently modulates gene expression. In the future, further investigations are necessary toward resolving whether $\alpha$-taxilin as well as $\gamma$-taxilin/FIAT is present in the nucleus and implicated in a transcription process.

In conclusion, our results indicate that $\alpha$-taxilin is localized on some intracellular components as a peripheral membrane protein and suggest that $\alpha$-taxilin functions as a linker protein in the association of the $\alpha$-taxilin-containing intracellular components with the microtubule cytoskeleton. Furthermore, our data provide an insight into the function of $\alpha$-taxilin, that is, $\alpha$-taxilin might be involved in other cellular functions in the nucleus other than intracellular vesicle traffic.

Acknowledgments. We thank Mayumi Ohshima and Akemi Akima for skillful assistance. This work was supported by Grants-in-Aid for scientific research from Ministry of Education, Culture, Sports, Science and Technology, Japan (2011).

\section{References}

Band, A.M., Maatta, J., Kaariainen, L., and Kuismanen, E. 2001. Inhibition of the membrane fusion machinery prevents exit from the TGN and proteolytic processing by furin. FEBS Lett., 505: 118-124.

Band, A.M., Ali, H., Vartiainen, M.K., Welti, S., Lappalainen, P., Olkkonen, V.M., and Kuismanen, E. 2002. Endogenous plasma membrane t-SNARE syntaxin 4 is present in rab11 positive endosomal membranes and associates with cortical actin cytoskeleton. FEBS Lett., 531: 513-519.

Band, A.M. and Kuismanen, E. 2005. Localization of plasma membrane t-SNAREs syntaxin 2 and 3 in intracellular compartments. BMC Cell Biol., 6: 26.

Bascom, J.L., Fata, J.E., Hirai, Y., Sternlicht, M.D., and Bissell, M.J. 2005. Epimorphin overexpression in the mouse mammary gland promotes alveolar hyperplasia and mammary adenocarcinoma. Cancer Res., 65: 8617-8621.

Behnia, R. and Munro, S. 2005. Organelle identity and the signposts for membrane traffic. Nature, 438: 597-604.
Bennett, M.K., Garcia-Arraras, J.E., Elferink, L.A., Peterson, K., Fleming, A.M., Hazuka, C.D., and Scheller, R.H. 1993. The syntaxin family of vesicular transport receptors. Cell, 74: 863-873.

Block, M.R., Glick, B.S., Wilcox, C.A., Wieland, F.T., and Rothman, J.E. 1988. Purification of an $N$-ethylmaleimide-sensitive protein catalyzing vesicular transport. Proc. Natl. Acad. Sci. USA, 85: 7852-7856.

Bradford, M.M. 1976. A rapid and sensitive method for the quantitation of microgram quantities of protein utilizing the principle of protein-dye binding. Anal. Biochem., 72: 248-254.

Brandt, R. and Lee, G. 1993. Functional organization of microtubuleassociated protein tau. Identification of regions which affect microtubule growth, nucleation, and bundle formation in vitro. J. Biol. Chem., 268: 3414-3419.

Brocker, C., Engelbrecht-Vandre, S., and Ungermann, C. 2010. Multisubunit tethering complexes and their role in membrane fusion. Curr. Biol., 20: R943-952.

Cai, H., Reinisch, K., and Ferro-Novick, S. 2007a. Coats, tethers, Rabs, and SNAREs work together to mediate the intracellular destination of a transport vesicle. Dev. Cell, 12: 671-682.

Cai, Q., Pan, P.Y., and Sheng, Z.H. 2007b. Syntabulin-kinesin-1 family member 5B-mediated axonal transport contributes to activity-dependent presynaptic assembly. J. Neurosci., 27: 7284-7296.

Chen, Y.A. and Scheller, R.H. 2001. SNARE-mediated membrane fusion. Nat. Rev. Mol. Cell Biol., 2: 98-106.

Fujiwara, T., Oda, K., Yokota, S., Takatsuki, A., and Ikehara, Y. 1988. Brefeldin A causes disassembly of the Golgi complex and accumulation of secretory proteins in the endoplasmic reticulum. J. Biol. Chem., 263: $18545-18552$.

Fujiwara, T., Yamamori, T., Yamaguchi, K., and Akagawa, K. 1997. Interaction of HPC-1/syntaxin 1A with the cytoskeletal protein, tubulin. Biochem. Biophys. Res. Commun., 231: 352-355.

Gillingham, A.K. and Munro, S. 2003. Long coiled-coil proteins and membrane traffic. Biochim. Biophys. Acta, 1641: 71-85.

Hashimoto, S., Onodera, Y., Hashimoto, A., Tanaka, M., Hamaguchi, M., Yamada, A., and Sabe, H. 2004. Requirement for Arf6 in breast cancer invasive activities. Proc. Natl. Acad. Sci. USA, 101: 6647-6652.

Helms, J.B. and Rothman, J.E. 1992. Inhibition by brefeldin A of a Golgi membrane enzyme that catalyses exchange of guanine nucleotide bound to ARF. Nature, 360: 352-354.

Honda, A., Yamada, M., Saisu, H., Takahashi, H., Mori, K.J., and Abe, T. 2002. Direct, $\mathrm{Ca}^{2+}$-dependent interaction between tubulin and synaptotagmin I: a possible mechanism for attaching synaptic vesicles to microtubules. J. Biol. Chem., 277: 20234-20242.

Iinuma, T., Aoki, T., Arasaki, K., Hirose, H., Yamamoto, A., Samata, R., Hauri, H-P., Arimitsu, N., Tagaya, M., and Tani, K. 2009. Role of syntaxin 18 in the organization of endoplasmic reticulum subdomains. J. Cell Sci., 122: 1680-1690.

Jahn, R. and Scheller, R.H. 2006. SNAREs-engines for membrane fusion. Nat. Rev. Mol. Cell Biol., 7: 631-643.

Kelly, R.B. 1990. Microtubules, membrane traffic, and cell organization. Cell, 61: 5-7.

Laemmli, U.K. 1970. Cleavage of structural proteins during the assembly of the head of bacteriophage T4. Nature, 227: 680-685.

Li, L., Omata, W., Kojima, I., and Shibata, H. 2001. Direct interaction of Rab4 with syntaxin 4. J. Biol. Chem., 276: 5265-5273.

Mashidori, T., Shirataki, H., Kamai, T., Nakamura, F., and Yoshida, K. 2011. Increased alpha-taxilin protein expression is associated with the metastatic and invasive potential of renal cell cancer. Biomed. Res., 32: $103-110$

Miyata, T., Ohnishi, H., Suzuki, J., Yoshikumi, Y., Ohno, H., Mashima, H., Yasuda, H., Ishijima, T., Osawa, H., Satoh, K., Sunada, K., Kita, H., Yamamoto, H., and Sugano, K. 2004. Involvement of syntaxin 4 in the transport of membrane-type 1 matrix metalloproteinase to the plasma 
membrane in human gastric epithelial cells. Biochem. Biophys. Res. Commun., 323: 118-124.

Nogami, S., Satoh, S., Nakano, M., Shimizu, H., Fukushima, H., Maruyama, A., Terano, A., and Shirataki, H. 2003a. Taxilin; a novel syntaxin-binding protein that is involved in $\mathrm{Ca}^{2+}$-dependent exocytosis in neuroendocrine cells. Genes Cells, 8: 17-28.

Nogami, S., Satoh, S., Nakano, M., Terano, A., and Shirataki, H. 2003b. Interaction of taxilin with syntaxin which does not form the SNARE complex. Biochem. Biophys. Res. Commun., 311: 797-802.

Nogami, S., Satoh, S., Tanaka-Nakadate, S., Yoshida, K., Nakano, M., Terano, A., and Shirataki, H. 2004. Identification and characterization of taxilin isoforms. Biochem. Biophys. Res. Commun., 319: 936-943.

Ohtomo, N., Tomiya, T., Tanoue, Y., Inoue, Y., Nishikawa, T., Ikeda, H., Seyama, Y., Kokudo, N., Shibahara, J., Fukayama, M., Koike, K., Shirataki, H., and Fujiwara, K. 2010. Expression of alpha-taxilin in hepatocellular carcinoma correlates with growth activity and malignant potential of the tumor. Int. J. Oncol., 37: 1417-1423.

Perez, F., Diamantopoulos, G.S., Stalder, R., and Kreis, T.E. 1999. CLIP170 highlights growing microtubule ends in vivo. Cell, 96: 517-527.

Phelps, K.K. and Walker, R.A. 2000. NEM tubulin inhibits microtubule minus end assembly by a reversible capping mechanism. Biochemistry, 39: $3877-3885$.

Pierre, P., Scheel, J., Rickard, J.E., and Kreis, T.E. 1992. CLIP-170 links endocytic vesicles to microtubules. Cell, 70: 887-900.

Rospert, S., Dubaquie, Y., and Gautschi, M. 2002. Nascent-polypeptideassociated complex. Cell. Mol. Life Sci., 59: 1632-1639.

Rothman, J.E. 1994. Mechanisms of intracellular protein transport. Nature, 372: 55-63.

Sapperstein, S.K., Walter, D.M., Grosvenor, A.R., Heuser, J.E., and Waters, M.G. 1995. p115 is a general vesicular transport factor related to the yeast endoplasmic reticulum to Golgi transport factor Usolp. Proc. Natl. Acad. Sci. USA, 92: 522-526.

Severin, F.F., Shanina, N.A., Kuznetsov, S.A., and Gelfand, V.I. 1991. MAP2-mediated binding of chromaffin granules to microtubules. FEBS
Lett., 282: 65-68.

Shorter, J., Beard, M.B., Seemann, J., Dirac-Svejstrup, A.B., and Warren, G. 2002. Sequential tethering of Golgins and catalysis of SNAREpin assembly by the vesicle-tethering protein p115. J. Cell Biol., 157: 45-62.

Steinmetz, M.O. and Akhmanova, A. 2008. Capturing protein tails by CAP-Gly domains. Trends Biochem. Sci., 33: 535-545.

Stromberg, S., Agnarsdottir, M., Magnusson, K., Rexhepaj, E., Bolander, A., Lundberg, E., Asplund, A., Ryan, D., Rafferty, M., Gallagher, W.M., Uhlen, M., Bergqvist, M., and Ponten, F. 2009. Selective expression of Syntaxin-7 protein in benign melanocytes and malignant melanoma. $J$. Proteome Res., 8: 1639-1646.

Su, Q., Cai, Q., Gerwin, C., Smith, C.L., and Sheng, Z.H. 2004. Syntabulin is a microtubule-associated protein implicated in syntaxin transport in neurons. Nat. Cell Biol., 6: 941-953.

Sztul, E. and Lupashin, V. 2006. Role of tethering factors in secretory membrane traffic. Am. J. Physiol. Cell Physiol., 290: C11-26.

Wang, S., Liu, Y., Adamson, C.L., Valdez, G., Guo, W., and Hsu, S.C. 2004. The mammalian exocyst, a complex required for exocytosis, inhibits tubulin polymerization. J. Biol. Chem., 279: 35958-35966.

Yoshida, K., Nogami, S., Satoh, S., Tanaka-Nakadate, S., Hiraishi, H., Terano, A., and Shirataki, H. 2005. Interaction of the taxilin family with the nascent polypeptide-associated complex that is involved in the transcriptional and translational processes. Genes Cells, 10: 465-476.

Yu, V.W., Ambartsoumian, G., Verlinden, L., Moir, J.M., Prud'homme, J., Gauthier, C., Roughley, P.J., and St-Arnaud, R. 2005. FIAT represses ATF4-mediated transcription to regulate bone mass in transgenic mice. J. Cell Biol., 169: 591-601.

Yu, V.W., Gauthier, C., and St-Arnaud, R. 2006. Inhibition of ATF4 transcriptional activity by FIAT/gamma-taxilin modulates bone mass accrual. Ann. N.Y. Acad. Sci., 1068: 131-142.

(Received for publication, February 2, 2012, accepted, June 5, 2012 and published online, June 16, 2012) 